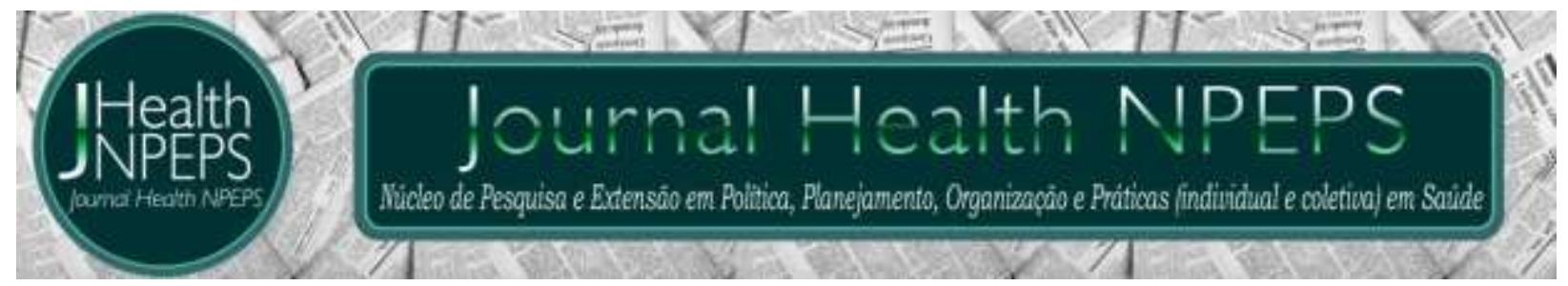

http://dx.doi.org/10.30681/252610103706

ARTIGO ORIGINAL

\title{
Significado atribuído por idosos com hipertensão arterial sistêmica à realização de atividade física
}

\section{Meaning attributed by elderly people with systemic arterial hypertension to physical activity}

\section{Significado atribuido por ancianos con hipertensión arterial sistémica la realización de actividad física}

\section{Alice Regina Costa Barbosa ${ }^{1}$, Bianca de Moura Peloso Carvalho², Camila Maria Silva Paraizo ${ }^{3}$, Eliza Maria Rezende Dázio ${ }^{4}$, Rogerio Silva Lima ${ }^{5}$, Silvana Maria Coelho Leite Fava ${ }^{6}$}

\section{RESUMO}

Objetivo: analisar o significado atribuído pelas pessoas idosas com hipertensão arterial sistêmica à atividade física. Método: pesquisa qualitativa, descritiva e exploratória, com idosos de uma unidade de estratégia de saúde da família de um município de Minas Gerais, em 2017. Os dados foram coletados por meio de entrevista semiestruturada, organizados e interpretados por meio de análise de conteúdo temática. Da análise, foi possível organizar o material empírico em

\footnotetext{
${ }^{1}$ Enfermeira. Mestranda do Programa de Pós Graduação em Enfermagem pela Universidade Federal de Alfenas (UNIFAL). Alfenas, Minas Gerais, Brasil. E-mail: reginalice25@gmail.com ORCID ID: https://orcid.org/00000002-5928-8411

${ }^{2}$ Enfermeira. Mestranda do Programa de Pós Graduação em Enfermagem pela Universidade Federal de Alfenas (UNIFAL). Alfenas, Minas Gerais, Brasil. E-mail: biancampcar@gmail.com ORCID ID: https://orcid.org/00000001-5336-2249

${ }^{3}$ Enfermeira. Doutoranda em Ciências pelo Programa de Pós-Graduação em Enfermagem Fundamental da Escola de Enfermagem de Ribeirão Preto da Universidade de São Paulo (EERP/USP). Ribeirão Preto, São Paulo, Brasil. E-mail:camila-maria88@hotmail.com ORCID ID: https://orcid.org/0000-0002-3574-7361

${ }^{4}$ Enfermeira. Doutora em Enfermagem. Docente da Escola de Enfermagem e do Programa de Pós-graduação em Enfermagem da Universidade Federal de Alfenas (UNIFAL). Alfenas, Minas Gerais, Brasil. E-mail: eliza.dazio@unifal-mg.edu.br ORCID ID: https://orcid.org/0000-0001-9216-6283.

${ }^{5}$ Enfermeiro. Doutor em Ciências. Docente da Escola de Enfermagem e do Curso de Medicina da Universidade Federal de Alfenas (UNIFAL). Alfenas, Minas Gerais, Brasil. E-mail: rogerio.lima@unifal-mg.edu.br ORCID ID: https://orcid.org/0000-0002-1751-2913

${ }^{6}$ Enfermeira. Doutora em Ciências. Docente da Escola de Enfermagem e do Programa de Pós Graduação em Enfermagem da Universidade Federal de Alfenas (UNIFAL). Alfenas, Minas Gerais, Brasil. E-mail: silvana.fava@unifal-mg.edu.br ORCID ID: https://orcid.org/0000-0003-3186-9596
}

Autor principal - Endereço para correspondência: Rua Gabriel Monteiro da Silva, 700 CEP: 37130001, AlfenasMG, Brasil.

Este artigo está licenciado sob forma de uma licença Creative Commons Atribuição 4.0 Internacional, que permite uso irrestrito, distribuição e reprodução em qualquer meio, desde que a publicação original seja corretamente citada. 
quatro categorias: motivos para realizar a atividade física, significado da atividade física, mudanças percebidas no cotidiano e percepção dos efeitos da atividade física na pressão arterial. Resultados: para o grupo estudado, a atividade física significa um local para interação, que por vezes, é único espaço de socialização e melhora do seu bem-estar. Verificou-se também a influência do modelo biomédico em relação aos motivos para realizar a atividade física e o controle de sua saúde. Conclusão: o estudo aponta a necessidade dos profissionais considerarem a atividade física como uma terapêutica importante, capaz de contribuir para melhoria não somente de patologias físicas, mas de condições que afetam a saúde física e mental.

Descritores: Hipertensão; Atividade Motora; Enfermagem em Saúde Comunitária; Saúde do Idoso.

\section{ABSTRACT}

Objective: to analyze the meaning attributed by elderly people with Systemic Arterial Hypertension to physical activity. Method: qualitative, descriptive and exploratory research with elderly from a family health strategy unit of a city of Minas Gerais, in 2017. Data were collected through semi-structured interviews, organized and interpreted through thematic content analysis. From the analysis, it was possible to organize the empirical material into four categories: reasons for performing physical activity, meaning of physical activity, perceived changes in daily life and perception of the effects of physical activity on blood pressure. Results: for the group studied, physical activity means a place for interaction, which is sometimes the only space for socializing and improving their well-being. It was also verified the influence of the biomedical model in relation to the reasons to perform the physical activity and the control of their health. Conclusion: the study points to the need for professionals to consider physical activity as an important therapy, capable of contributing to the improvement not only of physical pathologies, but of conditions that affect physical and mental health.

Descriptors: Hypertension; Motor Activity; Community Health Nursing; Health of the Elderly.

\section{RESUMEN}

Objetivo: analizar el significado que las personas mayores con hipertensión arterial sistémica atribuyen a la actividad física. Método: investigación cualitativa, descriptiva y exploratoria con adultos mayores de una unidad de estrategia de salud familiar de un municipio de Minas Gerais, en 2017. Los datos se recopilaron mediante entrevistas semiestructuradas, organizadas e interpretadas mediante análisis de contenido temático. A partir del análisis, fue posible organizar el material empírico en cuatro categorías: razones para realizar actividad física, significado de la actividad física, cambios percibidos en la vida diaria y percepción de los efectos de la actividad física sobre la presión arterial. Resultados: para el grupo estudiado, la actividad física significa un lugar para la interacción, que a veces es el único espacio para socializar y mejorar su bienestar. También se verificó la influencia del modelo biomédico en las razones para realizar actividad física y controlar su salud. Conclusión: el estudio señala la necesidad de que los profesionales consideren la actividad física como una terapia 
importante, capaz de contribuir a la mejora no solo de las patologías físicas, sino también de las condiciones que afectan la salud física y mental.

Descriptores: Hipertensión; Actividad Motora; Enfermería en Salud Comunitaria; Salud de Los Ancianos.

\section{INTRODUÇÃO}

A hipertensão arterial sistêmica (HAS) é definida como uma condição crônica multifatorial caracterizada por elevação sustentada dos níveis pressóricos $\geq 140$ e/ou 90 mmHg. Segundo dados epidemiológicos no Brasil, esta condição atinge 36 milhões de pessoas adultas, sendo que mais de $60 \%$ dessas são idosas ${ }^{1}$. A HAS relaciona-se às doenças cardiovasculares e seus agravos, que são as principais causas de óbitos no país, portanto é considerada um dos maiores problemas de saúde pública da atualidade ${ }^{2}$.

$$
\text { Em virtude disso, }
$$

intervenções na direção de diagnosticar, prevenir e tratar a HAS são de suma importância, e diversas estratégias alternativas no seu tratamento têm sido sugeridas ${ }^{3}$.

$$
0 \text { tratamento farmacológico }
$$
consiste no uso contínuo de antihipertensivos e o tratamento não farmacológico está relacionado à modificação do estilo de vida. A este respeito, há recomendação de que sejam adotadas uma alimentação saudável, diminuição da gordura corporal e, principalmente, a prática regular de exercícios físicos ${ }^{4}$.

Nesta direção, o exercício físico tem sido reconhecido como um dos métodos não farmacológicos mais eficientes para combater a $\mathrm{HAS}^{3}$. A prática regular de exercícios físicos possui baixo custo e risco mínimo, tendo como benefícios a diminuição ou controle dos níveis pressóricos, contribuindo também para a melhoria de outras doenças como, diabetes mellitus, dislipidemias e o controle do estresse, mal estar e insonia 4 .

Desse modo, a prática de atividade física tende a minimizar em até $60 \%$ os riscos cardiovasculares, assim como reduzir o uso de medicamentos antihipertensivos e gastos com o tratamento ${ }^{2,4}$. No entanto, estudos descrevem a baixa adesão à prática de atividade física por parte das pessoas com $\mathrm{HAS}^{6,7}$. 
As dificuldades de incorporar as práticas de atividade física no cotidiano podem estar relacionadas à falta de motivação para realizar essas atividades e ao significado a elas atribuído, influenciado pelas atitudes, valores e crenças. Estudos que buscam conhecer os significados da atividade física para pessoas com HAS, são escassos ${ }^{7,8}$ embora sejam fundamentais para o planejamento das ações de prevenção e promoção à saúde.

Diante disso, alguns questionamentos foram levantados: o que leva a pessoa idosa a participar do grupo de atividade física? 0 que significa para a pessoa idosa realizar atividades físicas neste grupo? A partir destas indagações, este estudo buscou analisar o significado atribuído pelas pessoas idosas com HAS à atividade física.

\section{MÉTODO}

\section{Trata-se de estudo} qualitativo, descritivo e exploratório, realizado com 15 idosos hipertensos, cadastrados em uma Estratégia Saúde da Família (ESF), situada em município do sul de Minas Gerais, no período de julho a agosto de 2017. A escolha pela unidade deu-se em razão de que nesta área de abrangência 0 grupo de atividade física ocorre regularmente.

Como critérios de inclusão, os participantes deveriam ser cadastrados na ESF, vinculado ao grupo de atividade física e apresentarem assiduidade nas atividades do grupo. Foram exclusos aqueles que não apresentaram diagnóstico de HAS.

Para a coleta de dados, optou-se pela técnica de entrevista semiestruturada. 0 roteiro utilizado foi constituído por duas partes: a primeira com o objetivo de identificar a caracterização sociodemográfica e clínica com as seguintes variáveis: gênero, idade, estado civil, escolaridade, crença religiosa, hábitos de vida (tabagismo e consumo de bebida alcoólica), tempo de participação no grupo, tempo de diagnóstico da HAS, comorbidades associadas, tipo de tratamento. A segunda parte foi constituída pelas seguintes questões norteadoras: 0 que leva você a participar do grupo de atividade física? 0 que significa para você 
realizar as atividades físicas neste grupo? Você percebeu alguma mudança na sua vida após a participação nas atividades físicas? Que mudanças foram estas? E quanto a sua pressão, houve alguma alteração?

As entrevistas ocorreram no domicílio do participante, após agendamento prévio. Foram registradas por meio de gravador digital e transcritas na integra pelo próprio pesquisador imediatamente após as gravações, com o auxílio do editor de texto. A duração de cada entrevista foi de aproximadamente 60 minutos.

Para análise dos dados, utilizou-se a análise de conteúdo temática9 ${ }^{9}$ Inicialmente realizou-se a transcrição integral do material coletado, leitura e releitura, em seguida a ordenação das falas. Foram selecionadas as ideias relevantes que constituem as unidades de significado, as quais foram codificadas, agrupadas de acordo com a sua similaridade e organizadas em categorias.

Este estudo é um recorte do projeto intitulado: "Planejamento estratégico e o papel da equipe da unidade de estratégia de saúde da família na promoção da saúde de pessoas com hipertensão arterial sistêmica". Obteve-se aprovação pelo Comitê de Ética em Pesquisa da Universidade Federal de Alfenas sob CAEE: 41227215.2 .0000 .5142 e parecer $n^{\circ} .91934$.

Os participantes foram esclarecidos sobre os objetivos da pesquisa, concederam a anuência em integrar o estudo por meio da assinatura do Termo de Consentimento Livre e Esclarecido (TCLE). Para garantia do anonimato dos participantes, atribuiu- se a letra "P" seguida de um algarismo arábico para identificação dos fragmentos de depoimentos.

\section{RESULTADOS E DISCUSSÃO}

Participaram do estudo 15 pessoas, com predomínio de mulheres $(93,33 \%)$, viúvas $(40 \%)$, com a média de idade de 64,6 anos, católica $(80 \%)$, com até oito anos de estudo $(64,28 \%)$.

0 predomínio do gênero feminino pode estar relacionado ao fato que a busca pela assistência em saúde é maior neste grupo 
populacional, isto, por sua vez, pode favorecer com que HAS ser mais diagnosticada neste extrato ${ }^{10,11}$. No que concerne à faixa etária, existe uma relação direta e linear entre envelhecer e HAS, associada à mudança epidemiológica e demográfica, com mais pessoas idosas no país e maior expectativa de vida ${ }^{1}$. Nesse contexto, a prevalência de HAS na população adulta brasileira, tende a representatividade de $50 \%$ em indivíduos com 60 a 69 anos e $75 \%$ entre os com mais de 70 anos $^{12}$.

Em relação aos hábitos de vida nenhum participante era tabagista, e apenas quatro relataram fazer uso de bebida alcoólica.

0 tempo médio de diagnóstico da HAS foi de 11,2 anos e 3,8 anos de participação no grupo. Em relação às comorbidades associadas, a mais citada foi Diabetes Mellitus (28,57\%).

As medicações mais utilizadas foram 0 hipotensor arterial, Losartana Potássica de 50mg e $25 \mathrm{mg}$ e o diurético depletor de potássio Hidroclorotiazida de 25mg.

\section{Motivos para realizar atividade física}

A atividade física configura-se para os participantes uma ação terapêutica para o alívio da dor e redução do peso.

É porque eu estava obesa... e dor muscular, dor nas juntas, aí a médica que atendia aqui no postinho foi quem disse pra mim. (P12)

O médico que me indicou, falou que a atividade física melhora tudo. (P9)

Eu voltei e parou de doer, quer dizer, eu não posso parar (P6)

Eu tinha muita dor, uma dor no joelho, quando eu ia subir morro me dava uma canseira, eu quase não aguentava. (P14)

A dor é uma das principais causas do sofrimento humano, ocasionando incapacidades, comprometendo a qualidade de vida e gerando imensuráveis repercussões na vida da pessoa $^{13}$. Pode ser considerada uma experiência sensorial e emocional desagradável que envolve mecanismos culturais, psíquicos, físicos, emocionais, cognitivos, neurológicos e sociais. Sendo uma condição de incomodo, todo ser humano procura evitar e que deve ser prontamente combatida, independentemente de suas convicções ${ }^{14,15}$. Daí que se pode entender que o alívio da dor se 
desponte como principal motivo pela busca para realização de atividades físicas.

Percebe-se que a dor incomoda a pessoa idosa porque se traduz pela manifestação corporal, enquanto a HAS pelo seu caráter silencioso e não sintomático não representa a prioridade para 0 cuidado/tratamento ${ }^{11}$.

Um estudo realizado em Belo Horizonte (MG) com pessoas com HAS apontou a existência de uma falsa ideia, entre os participantes, de que se não há presença de sintomas não faz sentido manter uma dieta hipossódica, deixar a bebida alcoólica e fazer atividade física. Nesta direção, ressalta-se que as melhores estratégias para o manejo da hipertensão arterial, possuem relação com o conhecimento das pessoas sobre a sua doença, visto que a condição crônica é um fenômeno complexo e multideterminado ${ }^{16}$.

Assim, há necessidade de que seja instaurado um processo educativo permanente junto a essas pessoas com HAS, oferecendo-lhes instrumentos para que reconheçam a doença e entendam a necessidade e importância da adesão ao tratamento ${ }^{17}$

\section{Significado da atividade física}

É sabido que um dos benefícios de realizar exercícios físicos é a melhoria da hipertensão arterial, com diminuição e/ou controle dos valores pressóricos ${ }^{18}$.

Contudo, o que precisa ser tomado em consideração é a premissa de que os significados para a participação das pessoas no grupo de atividade física envolvem respostas psicossociais, contextualizadas em um espaço de interação com os amigos, como se observa nos depoimentos.

É importantíssimo para mim fazer atividade física, [...] é sai de casa, encontra com outras pessoas, bate um papinho, sempre tem uma notícia, uma coisa, porque ficar só dentro de casa não é fácil não, interage com turma [...]. (P7)

[...] me sinto bem, parece que a gente sente mais ânimo né? [...] quando chega lá a gente anima, é bom, ver o povo tudo animado, dá vida pra gente, vê umas velhinhas tudo animado, a gente tem que ser assim, é melhor, é bem gostoso, eu gosto por tudo, pela atividade, por estar no meio do povo, junto, interagindo. (P11)

Em face dos resultados, em consonância ao encontrado na literatura, é possível inferir que a 
prática da atividade física, além de ser benéfica para as condições físicas do idoso, proporciona também efeitos positivos referentes ao perfil de humor das pessoas ${ }^{19,20}$. Influencia de forma significativa a qualidade de vida, principalmente dos idosos, que se sentem mais dispostos e alegres 8,21 .

0 exercício físico regular motiva as pessoas a adotarem melhores hábitos e é uma alternativa de tratamento para estresse, o que tem impactos favoráveis sobre todos os aspectos da vida ${ }^{21}$.

Estudo realizado no extremo sul do Brasil demonstrou que à medida que a frequência de atividade física aumenta, diminui a prevalência dos sintomas depressivos. Deste modo, quanto mais ativo for o idoso, maior será a tendência para este enfrentar as modificações que acompanham o envelhecimento de forma positiva e preservar 0 seu bem-estar psicológico ${ }^{22}$.

Nesta ótica, estudo realizado em Minas Gerais mostrou melhora da qualidade de vida em relação à capacidade funcional, recuperação geral da saúde, saúde mental, aspectos sociais e o bem estar físico, dos idosos que participavam de um grupo, em comparação com aqueles que não participavam ${ }^{23}$.

\section{Mudanças percebidas no cotidiano}

Percebe-se também, que a prática da atividade física para os idosos do estudo, representa uma busca por felicidade. Assim, sentir-se feliz e com mais ânimo são indicadores das principais modificações geradas com as atividades físicas.

\begin{abstract}
Melhora para dormir, melhora estresse do dia a dia, tudo ajuda, interage. (P3)

Ela é ótima pra gente, então, porque melhora o alto astral da gente em tudo, fico mais feliz, além de ser bom para a gente a gente tem amizade, e hoje em dia a pessoa ter amizade é muito bom, eu graças a Deus tenho umas veiaradinha muito boa na minha amizade. (P6)
\end{abstract}

Como observado, a atividade física se mostra como uma distração e uma forma de causar bem-estar e aumentando a autoestima ${ }^{24}$. E ao promover o bem-estar, há redução da ansiedade, efeitos nocivos do estresse e sintomas de depressão ${ }^{25}$.

0 estresse e a depressão configuram importantes problemas 
de saúde, visto que acometem mais de 300 milhões de pessoas no mundo ${ }^{26}$. Autores apontam que alterações emocionais, principalmente o estresse, podem ser fatores relacionados ao aumento do risco cardiovascular, facilitando o surgimento de emergências hipertensivas, lesões em órgãos alvo e agravamento de doenças cardiovasculares $^{11,26}$.

Outros autores enfatizam que - quadro de estresse interfere diretamente na elevação da pressão arterial, devido aos mecanismos fisiopatológicos que se associam ao estresse crônico e as alterações metabólicas. Deste entendimento, medidas como o exercício físico contribuem para prevenir, controlar e/ou evitar seu desenvolvimento ${ }^{27}$, promovendo um bem estar físico e mental ${ }^{28}$.

\section{Percepções dos efeitos da atividade física na pressão arterial}

Apreende-se que para alguns participantes, a atividade física tem pouca ação sob o controle dos níveis pressóricos e que esses são controlados apenas com 0 tratamento medicamento.
Sei lá, o povo fala que é bom fazer atividade, que serve como remédio. (P7)

Quanto à pressão não alterou em nada, porque eu tomo um monte de remédio para a pressão. (P8)

A visão curativa alicerça o entendimento das pessoas quanto às medidas não farmacológicas, como a atividade física. Talvez, essa concepção seja decorrente da forte influência do modelo assistencial medicalocêntrico sobre o modo de viver das pessoas ${ }^{29}$.

0 cuidado biomédico, centrado na doença, está presente em todos os níveis de assistência à saúde, como por exemplo, na Atenção Básica. Paradoxalmente, isto ocorre a despeito de que este nível de atenção, na história de sua constituição, tenha sido marcado por uma crítica ao paradigma mecanicista que fragmenta 0 atendimento a pessoa, com uso abusivo de tecnologias duras, relação verticalizada, impessoal e ações de caráter padronizado ${ }^{29}$.

0 enfrentamento de condições crônicas de saúde exige abordagens multidisciplinares e multiprofissionais, que impactem positivamente na redução dessas 
doenças e de seus fatores de risco ${ }^{19}$. Assim, entende-se que as propostas de promoção, assistência e reabilitação da saúde da pessoa idosa devem ir além do tratamento e controle de doenças específicas ${ }^{30}$. Além disso, essa forma de agir não contribui com a consolidação dos princípios que direcionam as ações da ESF e do Sistema Único de Saúde (SUS) porque estimulam um conjunto de crenças de que a busca pelo sistema de saúde deve ocorrer somente na presença de um agravo, fortalecendo assim, a visão social de dependência de atos prescritivos e medicamentosos ${ }^{31,32}$.

\section{CONSIDERAÇÕES FINAIS}

0 estudo identificou que os significados elaborados pelas pessoas idosas com relação às atividades físicas para controle da HAS são influenciados pelo modelo biomédico, hegemônico no contexto dos serviços de saúde no cenário nacional. Assim, o motivo da prática de atividade física e as mudanças percebidas em relação aos níveis pressóricos, na percepção dos idosos participantes, seguem a lógica da busca por alívio de sintomas álgicos e da ideia de que tal atividade não interfere no controle da PA.

0 estudo aponta a necessidade dos profissionais em considerarem a atividade física como uma terapêutica importante, capaz de facilitar as interações sociais e contribuir para melhoria não somente de patologias físicas, mas das condições que afetam a saúde mental. Considera-se que este estudo tem como limitação o fato de ter sido realizado com apenas uma técnica de coleta e com pequena amostra. Sugere-se assim, novos estudos com esse perfil populacional, na perspectiva de contribuir com a qualidade de vida no processo de envelhecimento.

\section{REFERENCIAS}

1. Malachias MVB, Póvoa RMS, Nogueira AR, Souza D, Costa LS, Magalhães ME. $7^{\text {a }}$ Diretriz Brasileira de Hipertensão Arterial: Capítulo 3 - Avaliação Clínica e Complementar. Arq Bras Cardiol. 2016; 107(supl. 3): 14-17.

2. Hortencio MNS, Silva JK, Zonta MA, Melo CPA, França CN. Efeitos de 
exercícios físicos sobre fatores de risco cardiovascular em idosos hipertensos. Rev Bras Prom Saúd. 2018; 31(2):1-9.

3. Neto Corrêa VG, Palma A. Impacto da atividade física na hipertensão arterial em adolescentes com sobrepeso e obesidade. ConScientiae Saúde. 2015; 14(1):32-39.

4. Kneubuehler PA, Mueller D. Aplicação e análise dos efeitos de sessões de exercício físico aeróbico e de resistência aplicada na academia ao ar livre no controle da hipertensão arterial. Rev Bras Presc Fisiol Exerc. $\quad$ 2016; 10(61):663-669.

5. Pierin AMG, Silva SSBE, Colosimo FC, Toma GA, Serafim TS, Meneghin P. Chronic and asymptomatic diseases influence the control of hypertension treatment in primary care. Rev esc enferm USP. 2016; 50(5):763-770.

6. Moura AA, Godoy S, Cesarino CB, Mendes IAC. Fatores da não adesão ao tratamento da hipertensão arterial. Enferm Glob. 2016; (43):14-27.

7. Oliveira-Filho AD, Leandro AKC, Almeida MQ, Junior Lyra DP, Neves
SJF. Hipertensão pseudo-resistente causada por baixa adesão terapêutica. Rev Ciênc Farm Básica Apl. 2015; 36(1):97-102.

8. Santos GLA, Santana RF, Broca PV. Execution capacity of instrumental activities of daily living in elderly: Ethnonursing. Esc Anna Nery Enferm. 2016; 20(3): e20160064.

9. Bardin L. Análise de Conteúdo. Lisboa: Edições 70; 2013.

10. Andrade SSCDA, Malta DC, Iser BM, Sampaio PC, Moura LD. Prevalência da hipertensão arterial autorreferida nas capitais brasileiras em 2011 e análise de sua tendência no período de 2006 a 2011. Rev bras epidemol. 2014; 17(1): 215-226.

11. Mendes FA, Silva MP, Ferreira CRS. Diagnósticos de enfermagem em portadores de hipertensão arterial na atenção primária. Estaç Cient (UNIFAP). 2018; 8(1):91-101.

12. Opas. Organização Pan-Americana da Saúde. Países das Américas apresentam benefícios de iniciativa para reduzir risco cardiovascular controlando a hipertensão. 2016. [acesso em 2016 Dez 4]. Disponível em: https://www.paho.org/bra/index. 
php?option=com_joomlabook\&vie $\mathrm{W}=$ topic\&id $=221$

13. Cunha CO, Pinto-Fiamengui LMS, Sampaio FA, Conti PCR. Is aerobic exercise useful to manage chronic pain? Rev dor. 2016; 17(1):61-4.

14. Almeida FC, Castilho A, Cesarino CB, Ribeiro RCHM, Martins MRI. Correlation between neuropathic pain and quality of life. BrJP. 2018; 1(4):349-53.

15. Ferretti F, Castanha AC, Padoan ER, Lutinski J, Silva MR. Quality of life in the elderly with and without chronic pain. BrJP. 2018; 1(2):111-5.

16. Silva LB, Soares SM, Silva PAB, Santos JFG, Miranda LCV, Santos RM. Assessment of the quality of primary care for the elderly according to the Chronic Care Model. Rev latinoam enferm. 2018; 26:e2987.

17. Dias EG, Souza ELS, Mishima SM. Contribuições da enfermagem na adesão ao tratamento da hipertensão arterial: uma revisão integrativa da literatura brasileira. Rev epidemiol controle infecç. 2016; 6(3):138-144.

18. Carvalho CJ, Marins JCB, Lande CG, Castilho PR, Reis HHT, Amrim
PRS, et al. Aerobic And Resistance Exercise In Patients With Resistant Hypertension. Rev bras med esporte. 2019; 25(2):107-111.

19. Araújo JTM, Rocha CF, Farias GMC, Cruz RS, Assunção Júnior JC, Silva HJA, et al. Experience with women with fibromyalgia who practice zumba. Case reports. Rev dor. 2017; 18(3):266-9.

20. Oliveira DV, Favero PF, Codonhato $\mathrm{R}$, Moreira CR, Antunes MD, Nascimento Júnior JRA. Investigação dos fatores psicológicos e emocionais de idosos frequentadores de clubes de dança de salão. Rev bras geriatr gerontol. 2017; 20(6):797804.

21. Sanches A, Costa R, Marcondes FK, Cunha TS. Relationship among stress, depression, cardiovascular and metabolic changes and physical exercise. Fisioter mov. 2016; 29(1):23-36.

22. Lange $C$, Heidemann ITSB, Castro DSP, Pinto AH, Peters C W, Durand MK. Promoting the autonomy of rural older adults in active aging. Rev bras enferm. 2018; 71(5):2411-7. 
23. Viana JV, Dias JMD, Batista PP, Silva SLA, Dias RC, Lustosa LP. Effect of a resistance exercise program for sarcopenic elderly women: quasi-experimental study. Fisioter mov. 2018; 31:e003111.

24. Geraldes AAR, Silva WB, Balikian Júnior PB, Pereira PMG. Effects of walking on the mood of physically active older people. Rev bras med esporte. 2019; 25(1):63-66.

25. Azofeifa Mora CAA. Revisión de los beneficios de la intensidad y modalidades del ejercicio físico sobre el estrés psicológico. Pensar em movimiento. 2018; 16(1):e30335.

26. OPAS. Organização Pan-Americana Da Saúde. Folha informativa Depressão. 2018 [citado em 2018 Mar 10]. Disponível em: https://www.paho.org/bra/index. php?option=com_content\&view=ar ticlectid=5635: folha-informativadepressao\&ltemid=1095.

27. Ferreira MM, Ferreira EF, Toledo C, Oliveira RAR. Relação da prática de atividade física $\mathrm{e}$ qualidade de vida na terceira idade. Cad cient FAGOC grad pósgrad. 2016; 1(2):9-15.
28. Silva PCS, Negrão MLB, Chini LT, Gonçalves MFC, Fava SMCL. Resignification of care for persons with systemic arterial hypertension. Rev enferm UFPE on line. 2018; 12(7): 1940-8.

29. Schenker M, Costa DHD. Advances and challenges of health care of the elderly population with chronic diseases in Primary Health Care. Ciênc Saúde Colet. 2019; 24(4):1369-1380.

30. Alves MB, Menezes MR, Felzemburg RDM, Silva VA, Amaral JB. Long-stay institutions for the elderly: physical-structural and organizational aspects. Esc Anna Nery Rev Enferm. 2017; 21(4):e20160337.

31. Amaral IT, Abrahão AL. Nursing consultation in Family Health Strategy, increasing the recognition of the distinct forms of action: an integrative review. J res: fundam care online. 2017; 9(4): 899-906.

32. Silva JMS, Bolpato MB. Principais causas de quedas em idosos e atuação da enfermagem nas orientações preventivas. J Health NPEPS. 2017; 2(2):418-429. 
Barbosa ARC, Carvalho BMP, Paraizo CMS, Dázio EMR, Lima RS, Fava SMCL. $\quad$ Significado atribuído por idosos...

Conflito de interesses: Os autores declaram não haver conflito de interesses.

\section{Participação dos autores:}

- Concepção: Barbosa ARC, Carvalho BMP, Paraizo CMS, Dázio EMR, Lima RS, Fava SMCL.

- Desenvolvimento: Barbosa ARC, Carvalho BMP, Paraizo CMS, Dázio EMR, Lima RS, Fava SMCL.

- Redação e revisão: Barbosa ARC, Carvalho BMP, Paraizo CMS, Dázio EMR, Lima RS, Fava SMCL.

Como citar este artigo: Barbosa ARC, Carvalho BMP, Paraizo CMS, Dázio EMR, Lima RS, Fava SMCL. Significado atribuído por idosos com hipertensão arterial sistêmica à realização de atividade física. J Health NPEPS. 2019; 4(2):90-103.

Submissão: 08/06/2019

Aceito: 02/10/2019

Publicado: 01/12/2019 